MAGNETOHYDRODYNAMICS Vol. 54 (2018), No. 1-2, pp. 153-156

DOI: $10.22364 / \mathrm{mhd} .54 .1-2.27$

\title{
DROP OF VISCOUS MAGNETIC FLUID IN A NON-STATIONARY MAGNETIC FIELD
}

\author{
A. Tyatyushkin \\ Institute of Mechanics, Moscow State University, \\ 1 Michurinskiy Ave., Moscow 119192, Russia
}

Small steady-state deformational oscillations of a drop of magnetic fluid in a nonstationary uniform magnetic field are theoretically investigated. The drop is suspended in a magnetic fluid immiscible with the drop fluid. The Reynolds number is so small that inertia can be neglected. The variation of the magnetic field is so slow that the approximation of quasi-stationary magnetic field and the approximation of quasi-steady flow may be used.

Introduction. A non-stationary magnetic field applied to a drop of a magnetic fluid suspended in an ordinary fluid or to a drop of an ordinary fluid suspended in a magnetic fluid causes a motion of the fluids inside and outside the drop. Investigation of this phenomenon is interesting from the point of view of its application to drive liquids into motion in various devices including microfluidic ones. Besides, the study of this phenomenon is interesting from the point of view of fundamental science.

Results of experimental investigations of the behavior of magnetic fluid drops are presented in a number of works (see [1] for references). For the theoretical description of the behavior of a magnetic fluid drop, models based on rather strong assumptions about the shape of the drop and about the flow inside and outside it were used [1]. The goal of the present study is to solve the problem of the drop shape and flow inside and outside it not using similar assumptions. With this approach, the shape of the drop is determined by equations derived with the help of asymptotic methods from the system of equations and boundary conditions which determine the magnetic field and the flow. In order to provide the applicability of the asymptotic methods, the present theoretical investigation is restricted to the case of weakly deformed drops.

\section{Problem formulation.}

1.1. Object of investigation. Let us consider a drop of an incompressible magnetic fluid exposed to a non-stationary uniform magnetic field with the intensity $\mathbf{H}_{\mathrm{a}}=\mathbf{H}_{\mathrm{a}}(t)$, where $t$ is the time. The viscosity and the magnetic permeability of the fluid inside the drop are $\eta_{\mathrm{i}}$ and $\mu_{\mathrm{i}}$. The radius of the undeformed drop is $a$. The drop is suspended in an incompressible magnetic fluid with the viscosity $\eta_{\mathrm{e}}$ and magnetic permeability $\mu_{\mathrm{e}}$. The surface tension of the interface between the fluids is $\sigma_{\mathrm{s}}$. The fluids are assumed to be sufficiently viscous so that the small Reynolds number approximation is valid. The variation of the magnetic field is so slow and the conductivities of the fluids are so small that the ferrohydrodynamic approximation is valid (see [2]).

1.2. System of equations. In order to determine the drop shape, the magnetic field intensity $\mathbf{H}$, the velocity $\mathbf{v}$ and the pressure $p$ as functions of the radiusvector $\mathbf{r}$ and time, under the above made assumptions, a system of equations of ferrohydrodynamics presented in a quasi-stationary magnetic field approximation and in a quasi-steady flow approximation is used. This system consists of the 


\section{A. Tyatyushkin}

continuity equation for incompressible fluid

$$
\nabla \cdot \mathbf{v}=0
$$

the motion equation in the approximations of small Reynolds number and quasisteady flow

$$
\nabla \cdot\left(-p \hat{I}+\hat{\sigma}_{\mathrm{m}}+\hat{\sigma}_{\mathrm{v}}\right)=0,
$$

the Maxwell's equations in the approximations of ferrohydrodynamics and quasistationary field

$$
\nabla \cdot \mathbf{B}=0, \quad \nabla \times \mathbf{H}=0,
$$

and the constitutive relation

$$
\mathbf{B}=\mu \mathbf{H} .
$$

Here, the formulas are expressed using the Gaussian system of units, '.' and ' $\times$ ' denote the scalar and vector products, $\mathbf{B}$ is the magnetic induction, $\eta=\eta_{\mathrm{i}}$ and $\mu=\mu_{\mathrm{i}}$ inside the drop, $\eta=\eta_{\mathrm{e}}$ and $\mu=\mu_{\mathrm{e}}$ outside it, $\nabla$ is the nabla operator, $\hat{I}$ is the identity tensor, $\hat{\sigma}_{\mathrm{m}}$ and $\hat{\sigma}_{\mathrm{v}}$ are the tensors of magnetic and viscous stresses expressed as

$$
\begin{aligned}
\hat{\sigma}_{\mathrm{m}} & =\frac{1}{4 \pi} \mathbf{B H}-\frac{1}{8 \pi} \mathbf{B} \cdot \mathbf{H} \hat{I}, \\
\hat{\sigma}_{\mathrm{v}} & =2 \eta(\nabla \mathbf{v})^{\mathrm{S}},
\end{aligned}
$$

where $\mathbf{A B}$ denotes the dyadic product of the vectors $\mathbf{A}$ and $\mathbf{B}, \nabla \mathbf{f}$ denotes the dyadic product of the nabla operator and the vector field $\mathbf{f}=\mathbf{f}(\mathbf{r}), \hat{T}^{\mathrm{S}}$ denotes the symmetric part of the tensor $\hat{T}$.

Using the continuity equation (1) and Maxwell's equations (3) and taking into account that the magnetic permeability is uniform, the motion equation (2) can be rewritten in the form of the Navier-Stokes equation in the approximation of small Reynolds number,

$$
-\nabla p+\eta \Delta \mathbf{v}=0
$$

where $\Delta$ is the Laplacian.

1.3. Boundary conditions. The boundary conditions at the boundary between the fluids include the condition of impenetrability,

$$
\left.\mathbf{v}\right|_{\mathrm{e}} \cdot \mathbf{n}=\left.\mathbf{v}\right|_{\mathrm{i}} \cdot \mathbf{n}=v_{\mathrm{s} n},
$$

the no-slip condition

$$
[\mathbf{v}]_{\mathrm{S}} \times \mathbf{n}=0,
$$

the conditions for the jumps of the normal and tangential components of the stress vector $\sigma_{n}=\mathbf{n} \cdot\left(-p \hat{I}+\hat{\sigma}_{\mathrm{m}}+\hat{\sigma}_{\mathrm{v}}\right)$

$$
\begin{aligned}
\mathbf{n} \cdot\left[-p \hat{I}+\hat{\sigma}_{\mathrm{m}}+\hat{\sigma}_{\mathrm{v}}\right]_{\mathrm{s}} \cdot \mathbf{n} & =-\sigma_{\mathrm{s}} \mathscr{H}, \\
\mathbf{n} \cdot\left[\hat{\sigma}_{\mathrm{m}}+\hat{\sigma}_{\mathrm{v}}\right]_{\mathrm{s}} \times \mathbf{n} & =0,
\end{aligned}
$$

the continuity conditions for the tangential component of the magnetic field intensity

$$
[\mathbf{H}]_{\mathrm{s}} \times \mathbf{n}=0,
$$

and for the normal component of the magnetic field induction

$$
[\mathbf{B}]_{\mathrm{S}} \cdot \mathbf{n}=0 .
$$




\section{Drop of viscous magnetic fluid in a non-stationary magnetic field}

Here, $\left.A\right|_{\mathrm{i}}$ and $\left.A\right|_{\mathrm{e}}$ are the values of the quantity $A$ at the boundary between the fluids from inside and outside the drop, respectively, $[A]_{\mathrm{s}}=\left.A\right|_{\mathrm{e}}-\left.A\right|_{\mathrm{i}}$ stands for the jump of the quantity $A$ at the boundary when moving from the inside to the outside, $\nabla_{\mathrm{s}}$ is the surface nabla operator, $\mathbf{n}$ is the external normal unit vector at a given point of the boundary, $v_{\mathrm{s} n}$ is the normal component of the velocity of the drop surface at a given point, $\mathscr{H}$ is the mean curvature at a given point of the drop surface. Note that the definition of the mean curvature used in the present work is such that it takes negative values on the surface of a convex domain.

The continuity conditions for the tangential components of the velocity and electric field intensity make it possible to determine the following vector fields defined on the drop surface

$$
\begin{aligned}
\mathbf{v}_{\mathrm{s} \tau} & =\mathbf{n} \times\left(\left.\mathbf{v}\right|_{\mathrm{i}} \times \mathbf{n}\right)=\mathbf{n} \times\left(\left.\mathbf{v}\right|_{\mathrm{e}} \times \mathbf{n}\right), \\
\mathbf{H}_{\mathrm{s}} & =\mathbf{n} \times\left(\left.\mathbf{H}\right|_{\mathrm{i}} \times \mathbf{n}\right)=\mathbf{n} \times\left(\left.\mathbf{H}\right|_{\mathrm{e}} \times \mathbf{n}\right) .
\end{aligned}
$$

The boundary conditions at infinity have the form

$$
\begin{aligned}
\mathbf{v} & \rightarrow 0 & \text { as } & r \rightarrow \infty, \\
p & \rightarrow p_{\infty} & \text { as } & r \rightarrow \infty, \\
\mathbf{H} & \rightarrow \mathbf{H}_{\mathrm{a}} & \text { as } & r \rightarrow \infty,
\end{aligned}
$$

where $p_{\infty}$ is the pressure at infinity. Besides, $\mathbf{v}(\mathbf{r}, t), p(\mathbf{r}, t)$ and $\mathbf{H}(\mathbf{r}, t)$ should be bounded for all bounded values of $\mathbf{r}$.

1.4. Drop deformation. Let the surface of the drop be given by the following equation

$$
r=|\mathbf{r}|=a+h\left(\frac{\mathbf{r}}{r}, t\right)
$$

In make the deformations of the drop small, the following condition should be fulfilled

$$
h=h\left(\frac{\mathbf{r}}{r}, t\right) \ll a .
$$

2. Solution. The presentation of the magnetic field intensity in the form of multipole expansion [3] and the general Lamb's solution [4] for the flow written in terms of the irreducible tensors [3] are used to solve the problem. With this approach, the magnetic field intensity, velocity and the pressure are sought for in the form of series with scalar, vector and tensor coefficients for which some relations are obtained that allow one to define these coefficients. With the use of these relations, the coefficients and the function $h=h(\mathbf{r} / r, t)$ are sought for in the form of asymptotic expansions over the parameter

$$
\alpha=\frac{9 a \mu_{\mathrm{e}} \max \left(H_{\mathrm{a}}\right)^{2}}{32 \pi \sigma_{\mathrm{s}}},
$$

the smallness of which provides the smallness of the drop deformations.

With an accuracy up to the terms of the first order, in an applied oscillating magnetic field with the intensity

$$
\mathbf{H}_{\mathrm{a}}=H_{\mathrm{am}} \cos (\omega t) \mathbf{k},
$$

the drop is a prolate spheroid with the axis directed along $\mathbf{k}$ and with the semiaxes

$$
\begin{array}{r}
a_{1}=a_{2}=a\left\{1-\frac{\alpha}{6}\left(\frac{\mu_{\mathrm{i}}-\mu_{\mathrm{e}}}{\mu_{\mathrm{i}}+2 \mu_{\mathrm{e}}}\right)^{2}\left[1+\frac{\cos (2 \omega t-2 \phi)}{\sqrt{1+4 \tau^{2} \omega^{2}}}\right]\right\}, \\
a_{3}=a\left\{1+\frac{\alpha}{3}\left(\frac{\mu_{\mathrm{i}}-\mu_{\mathrm{e}}}{\mu_{\mathrm{i}}+2 \mu_{\mathrm{e}}}\right)^{2}\left[1+\frac{\cos (2 \omega t-2 \phi)}{\sqrt{1+4 \tau^{2} \omega^{2}}}\right]\right\},
\end{array}
$$




\section{A. Tyatyushkin}

where

$$
\begin{gathered}
\tau=\frac{\left(16 \eta_{\mathrm{e}}+19 \eta_{\mathrm{i}}\right)\left(3 \eta_{\mathrm{e}}+2 \eta_{\mathrm{i}}\right)}{10\left(\eta_{\mathrm{e}}+\eta_{\mathrm{i}}\right)^{2}} \frac{a\left(\eta_{\mathrm{e}}+\eta_{\mathrm{i}}\right)}{4 \sigma_{\mathrm{s}}}, \\
\phi=\frac{1}{2} \arctan (2 \tau \omega) .
\end{gathered}
$$

Thus, the drop performs deformational oscillations with the angular frequency $2 \omega$ and the phase lag $2 \phi$.

In an applied rotating magnetic field with the intensity

$$
\mathbf{H}_{\mathrm{a}}=H_{\mathrm{am}}[\cos (\omega t) \mathbf{i}+\sin (\omega t) \mathbf{j}],
$$

the drop takes the shape of a tri-axial ellipsoid with the semiaxes

$$
\begin{aligned}
& a_{1}=a\left[1-\frac{\alpha}{3}\left(\frac{\mu_{\mathrm{i}}-\mu_{\mathrm{e}}}{\mu_{\mathrm{i}}+2 \mu_{\mathrm{e}}}\right)^{2}\right], \\
& a_{2}=a\left[1+\frac{\alpha}{6}\left(\frac{\mu_{\mathrm{i}}-\mu_{\mathrm{e}}}{\mu_{\mathrm{i}}+2 \mu_{\mathrm{e}}}\right)^{2}\left(1-\frac{3}{\sqrt{1+4 \tau^{2} \omega^{2}}}\right)\right], \\
& a_{3}=a\left[1+\frac{\alpha}{6}\left(\frac{\mu_{\mathrm{i}}-\mu_{\mathrm{e}}}{\mu_{\mathrm{i}}+2 \mu_{\mathrm{e}}}\right)^{2}\left(1+\frac{3}{\sqrt{1+4 \tau^{2} \omega^{2}}}\right)\right] .
\end{aligned}
$$

The ellipsoid rotates around its minor axis directed along $\mathbf{k}=\mathbf{i} \times \mathbf{j}$, with the angular velocity $\omega$ so that its major axis lags from $\mathbf{H}_{\mathrm{a}}$ by the angle $\phi$. Here, $\mathbf{i}, \mathbf{j}$ and $\mathbf{k}$ form a triple of orthonormal vectors.

Acknowledgements. Partial support by RFBR grants nos. 16-01-00157 and 17-01-00037 is acknowledged.

\section{References}

[1] A.V. Lebedev, A. Engel, K.I. Morozov and H. Bauke. Ferrofluid drops in rotating magnetic fields. New J. Phys., vol. 5 (2003), pp. 57.1-57.20.

[2] R.E. Rosensweig. Ferrohydrodynamics (Cambridge University Press, New York, 1985).

[3] L.D. Landau and E.M. Lifshits. The Classical Theory of Fields (Pergamon Press, 1975).

[4] H. Lamb. Hydrodynamics (Cambridge University Press, 1932).

Received 27.12.2017 